\title{
BEAM POSITION MONITOR SYSTEM FOR PEP-II
}

\author{
Stephen R. Smith, G. Roberto Aiello, Linda J. Hendrickson, Ronald G. Johnson, Mark R. Mills, and \\ Jeff J. Olsen, Stanford Linear Accelerator Center, Stanford, CA 94309, USA
}

\section{Abstract}

We describe the beam position monitor system built for PEP-II, the B-factory at SLAC. The system reports beam position for bunches of between $5 \times 10^{8}$ and $8 \times 10^{10}$ electron charges, either singly or as continuous streams of bunches every $4.2 \mathrm{~ns}$. Resolution at full charge is to be better than 10 microns in a single turn. Higher resolution is available via on-board multi-turn averaging. The position signal is processed in a $20 \mathrm{MHz}$ bandwidth around $952 \mathrm{MHz}$. This bandwidth, rather broader than that typical of RF position monitors, allows good resolution for low charge single bunches. Additional novel features include stringent control of return losses in order to minimize cross-talk between nearby bunches which may contain very different charges. The digitizing electronics is multiplexed between the two PEP-II storage rings. Design, construction, and installation experience, as well as first results with beam are presented.

\section{PEP-II}

The PEP-II B-Factory ${ }^{1}$ is based on colliding e+ and ebeams stored in two rings operating at different energies. Currently the high energy ring is nearly complete; the low energy ring will be installed later this year. Both rings will contain up to 1658 bunches of electrons or positrons. The high energy ring will store currents up to $1 \mathrm{Amp}$, while the low energy ring, to be installed later this year, will operate to $3 \mathrm{Amps}$. The machine is to operate as a factory, meaning that the machine is expected to be colliding efficiently a large fraction of the time; sufficient diagnostics must be available to keep it running optimally.

\begin{tabular}{|c|c|}
\hline Parameter & Value \\
\hline Position Monitors & 608 in two rings \\
\hline RF frequency & $476 \mathrm{MHz}$ \\
\hline Bunch spacing & $238 \mathrm{MHz}$ \\
\hline Filled bunches & 1658 \\
\hline Revolution Frequency & $136 \mathrm{kHz}$ \\
\hline BPM Processors & 170 \\
\hline Processing frequency & $952 \mathrm{MHz}$ \\
\hline Time resolution & $\sim 5 \mathrm{bunches}$ \\
\hline Measurement rate & $136 \mathrm{kHz}$ \\
\hline New measurement every & $120 \mathrm{~Hz}$ \\
\hline
\end{tabular}

Table 1. PEP-II and beam position monitor system parameters.

Challenging aspects for the BPM system are the need for high dynamic range, from $8 \times 10^{10} \mathrm{e}^{+}$per bunch at a 238
$\mathrm{MHz}$ rate, down to a single bunch of $5 \times 10^{8} \mathrm{e}$. Good resolution and stability are required, as is the ability to measure even low-charge single bunches injected into an $80 \mathrm{~ns}$ gap in an otherwise filled storage ring.

\begin{tabular}{|c|c|}
\hline Measurement Mode & Required Resolution $(\sigma)$ \\
\hline Single bunch, single turn & $1 \mathrm{~mm} @ 5 \times 10^{8} \mathrm{e}^{-}$ \\
\hline & and $100 \mu \mathrm{m} @ 1 \times 10^{10} \mathrm{e}^{-}$ \\
\hline Single bunch, $10^{3}$ turns & $15 \mu \mathrm{m} @ 1 \times 10^{10} \mathrm{e}^{-}$ \\
\hline Multi-bunch, single turn & $<10 \mu \mathrm{m} @ 1 \times 10^{10} \mathrm{e}^{-}$ \\
\hline
\end{tabular}

Table 2. BPM resolution requirements.

\section{BEAM POSITION TRANSDUCERS}

Each monitor consists of four buttons welded into the beam duct walls. Most BPM's have their button signals added pairwise so they read out either horizontal or vertical position. Buttons are $1.5 \mathrm{~cm}$ diameter in beam ducts with radii from $3.3 \mathrm{~cm}$ in the arc chambers to 4.5 $\mathrm{cm}$ in the straight sections. PEP-II RF is at $476 \mathrm{MHz}$. The first harmonic, $952 \mathrm{MHz}$ was chosen for the BPM processing frequency. The button transfer impedance was calulated with MAFIA, as were wake-field impedances due to the buttons. The latter led to slight redesign of the buttons to meet the wake-field budget consistant with providing sufficient signal to meet BPM resolution requirements at low current.

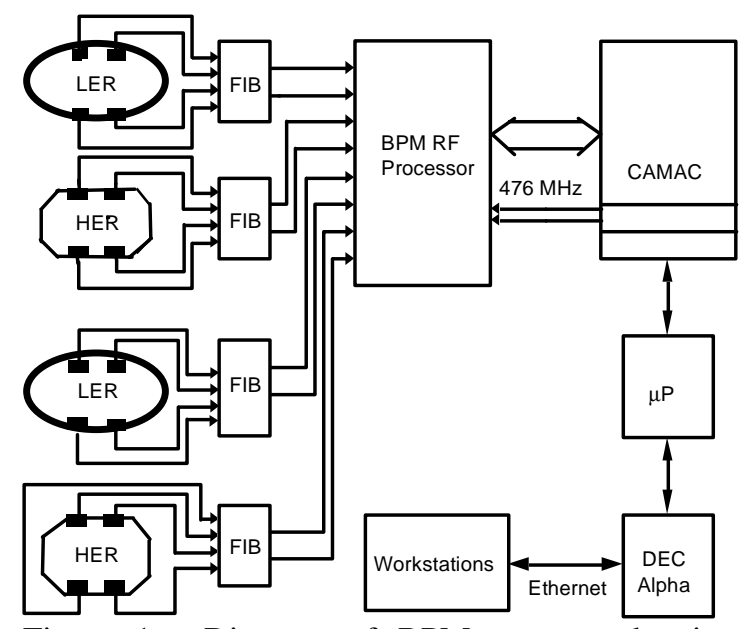

Figure 1. Diagram of BPM system, showing beam pickups, filter-isolator boxes, BPM processor, CAMAC, and control system. 


\section{FRONT-END ELECTRONICS}

Button signals are preprocessed in a "filter-isolator box" (FIB) mounted within a few feet of the buttons. This device is a constant input impedance $952 \mathrm{MHz}$ band-pass filter with an RF isolator on each output so that its outputs are well-matched to the $50 \Omega$ long-haul cables. Low $\mathrm{S}_{22}$ is needed to suppress round-trip reflections on the long-haul cable plant which would otherwise contaminate measurements of small single-bunches injected into a short gap in the high-current stored beam. The FIB sums the buttons pairwise for those BPM's where only a single coordinate is measured. It also provides a DC block and HV bias capability should we decide to place an ionclearing field at the button locations.

\section{DIGITIZING ELECTRONICS}

The choice of $952 \mathrm{MHz}$ as the processing frequency constrains the digitizing electronics to be located in the tunnel. Signal loss and cable costs prohibit bringing the signals out of the tunnel except for the BPM's closest to the centers of the straight sections. The processors are in CAMAC crates underneath the high energy ring bend magnets, which provide some shielding from synchrotron radiation.

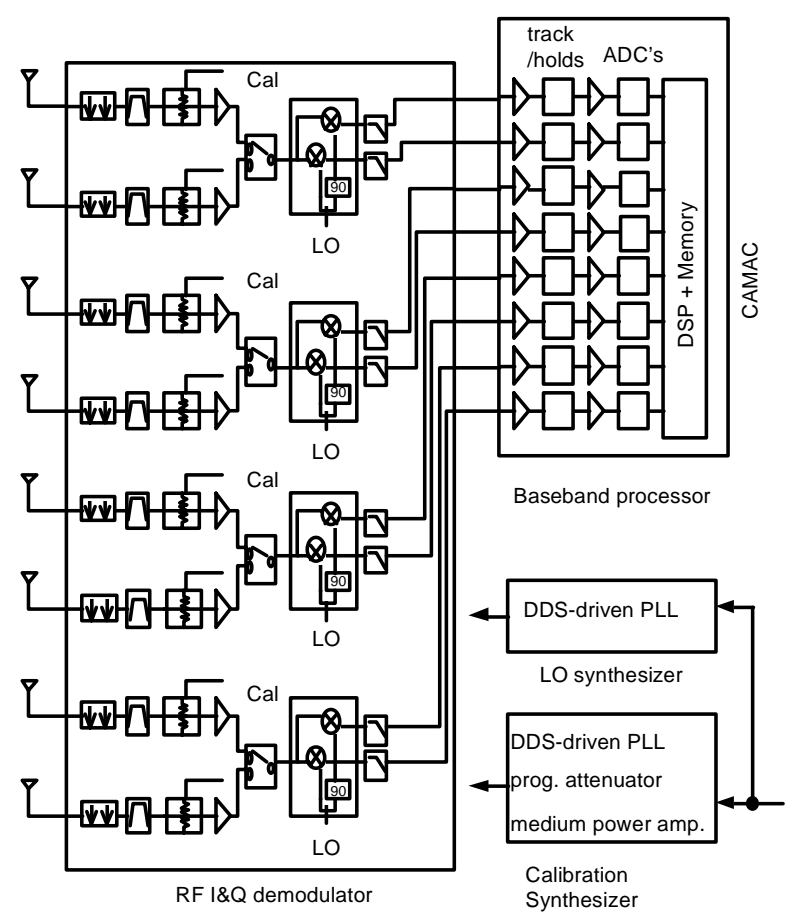

Figure 2. Ring I\&Q BPM Processor block diagram. Each measures an $\mathrm{X}$ and $\mathrm{Y}$ BPM for each of two rings.

Signal processing is based on direct quadrature (I\&Q) down-conversion of the $952 \mathrm{MHz}$ bunch harmonic pesent in the button spectrum. A $20 \mathrm{MHz}$ bandwidth was chosen to provide adequate signal-to-noise in the measurement of low-charge single bunches. The ratio of bandwidth to center frequency swayed the decision toward direct downconversion rather than the more conventional dual conversion via an intemediate frequency. Quadrature demodulation was chosen to elimate the need for either a stable phase reference or a PLL locked to the beam which might prove troublesome at low currents absent stored beam.

Each processor module handles BPM's from both storage rings, selectable by a programmable high-isolation switch. The processor signal path includes a calibration coupler, a band-pass filter, programmable attenuator, and low-noise preamplifier in front of the switch. The ringselection switch is followed by a quadrature demodulator which downconverts the $952 \mathrm{MHz}$ signal directly to baseband. Both the in-phase and quadrature components are low-pass-filtered, sampled, and digitized at the ring revolution frequency. A DSP converts eight digitized vector components to the four button amplitudes. Position is calculated from the ratios of the differences of the amplitudes divided by their sums. The DSP also computes multi-turn averages, if requested.

\section{CALIBRATION SCHEME}

Each measurement of an $\mathrm{X}, \mathrm{Y}$ position requires accurate measurement of the I and Q components of the signals on four buttons. Thus there are 8 voltages measured. Assuming linear response of the system, this requires knowledge of 18 calibration parameters: 8 pedestals (or ADC zero offsets), 4 ratios of the gains of in-phase channels to their respective quadrature channels, 4 I-to-Q phase angle errors, and the two ratios $\mathrm{A}_{\text {up }} / \mathrm{A}_{\text {down }}$ and $A_{\text {left }} / A_{\text {right }}$ which give the position offsets of a centered beam. These calibration parameters can be expected to drift with time and temperature and, in a factory machine which can't shut down to re-calibrate, must be measurable in the presence of stored beam.

Calibration tones, synthesised by an on-board DDSdriven PLL, are injected into the processor signal path upstream of the initial band-pass filter. Gain differences upstream of this point are expected to be static, and measured in the lab. The downconverted I \& Q components are digitzed at the ring-turn rate of $136 \mathrm{kHz}$ and fitted to a sine wave at the difference frequency between the local oscillator and the calibration tone. Calibration data is acquired during the ion-clearing gap. Beam-induced signal is further rejected by the fitting process when the the L.O. and calibration frequencies are chosen to be different from each other and from the 952 $\mathrm{MHz}$ harmonic of the bunch frequency. The amplitude, offset, and phase angles of the fitted sine curves provide all the needed calibration data.

A train of bunches at $4.2 \mathrm{~ns}$ spacing produces a line spectrum at the button, while a single bunch passing the button gives a spectrum broad compared to the bandwidth of the electronics. For bunch-train measurements 
the processor is calibrated at $952 \mathrm{MHZ}$, while for singlebunch measurements gains are averaged over the channel bandwidth.

\section{STATUS}

Checkout of the processing electronics in the tunnel indicate the resolution and accuracy requirements are met. Measurements of simulated beam show $10^{3}$ turn resolution $\sigma<1 \mu \mathrm{m}$ at moderate currents.

All high-energy ring BPM's are installed, except for those in the interaction region. First beam was injected into the high energy ring and transported to a temporary beam dump upstream of the interaction region on May 10.
The beam was seen on BPM's, but neither the beam nor the response of the position monitors to it are wellcharacterized at this time.

\section{ACKNOWLEDGEMENTS}

Work supported by the Department of Energy, contract DE-AC03-76SF00515.

\section{REFERENCES}

[1] PEP-II, An Asymmetric B Factory, Conceptual Design Report, SLAC-418, June 1993. 